\title{
Predictability of SST in a Stochastic Climate Model and Its Application to the Kuroshio Extension Region
}

\author{
ROBERT B. SCOTT \\ Institute for Geophysics, University of Texas at Austin, Austin, Texas \\ Bo QIU \\ School of Ocean and Earth Science and Technology, University of Hawaii at Manoa, Honolulu, Hawaii
}

(Manuscript received 12 October 2001, in final form 5 August 2002)

\begin{abstract}
The influence of deterministic forcing on SST predictability is investigated in a zero-dimensional, stochastic, coupled atmosphere-ocean climate model. The SST anomaly predictability time is found to be very sensitive to the properties of the deterministic forcing. Comparison of the amplitudes of the deterministic and stochastic forcing terms, for example, as determined from linear regression analysis, may give a misleading impression of their relative importance. The importance of instead comparing the time-integrated forcing terms is emphasized. The conditions under which the model exhibits preferred timescales and the conditions under which the model power spectrum approaches that of a univariate Markov process (red noise) are also determined.

The idealized model results are complemented with an analysis of climate observations for the Kuroshio Extension region. Observational errors and unresolved components of the enthalpy budget limited the maximum timescale considered to about $4 \mathrm{yr}$. This analysis revealed that the advection of anomalous geostrophic currents is a minor source of SST variability and not the limiting factor in determining SST predictability in that region, at least for the timescales considered.
\end{abstract}

\section{Introduction}

Although the possibility of seasonal forecasts of tropical climate variations associated with ENSO has been demonstrated (see e.g., Latif et al. 1998), the predictability of the extratropics remains an open question. It is clear that if midlatitude seasonal and longer timescale climate variations are predictable, it must be due to the dependence of the atmosphere on a slower component of the climate system. A likely candidate is the midlatitude sea surface temperature (SST), though other possibilities include a remote dependence upon polar sea ice (e.g., Yuan and Martison 2000), or tropical SST (reviewed by Alexander et al. 2002), or even radiative forcing associated with greenhouse gases. However, two major challenges to the midlatitude predictability problem persist. First the response of the atmosphere to midlatitude SST is complex and remains controversial (e.g, see reviews by Palmer 1996; Kushnir and Held 1996; Frankignoul 1985; Namias and Cayan 1981), though some encouraging results were found recently by Bond and Harrison (2000). Furthermore, the predictability of

Corresponding author address: Dr. Robert B. Scott, Institute for Geophysics, University of Texas at Austin, 4412 Spicewood Springs Rd., Building 600, Austin, TX 78759.

E-mail: rscott@ig.utexas.edu the SST remains to be established. This study addresses the latter challenge.

Observational studies, coupled AGCM-OGCM studies, and idealized modeling studies give conflicting results for the predictability of midlatitude SST anomalies. Two observational studies reveal propagating SST anomalies that persist for several years in the midlatitude North Atlantic (Hansen and Bezdek 1996; Sutton and Allen 1997). However, the leading EOFs of SST anomalies, which are constrained to be stationary patterns, have decay times of only about 5 months (Frankignoul 1985, Fig. 19). Namias and Cayan (1981) show that basin average SST anomalies in the North Pacific can persist for more than a decade.

Coupled atmosphere-ocean climate models generally, though not unanimously, suggest the leading modes of SST have predictability times of at least a few years. Using ensemble integrations of the Geophysical Fluid Dynamics Laboratory (GFDL) coupled atmosphereocean climate model, Griffies and Bryan (1997a,b) find predictability times of up to 5-7 yr for the dominant modes of SST variability in the North Atlantic. A recent update on this study using a higher-resolution version of the GFDL coupled model found even longer SST predictability times (S. Griffies, T. Delworth, R. Stouffer, G. Vallis 2001, personal communication). These re- 
sults are consistent with those of Saravanan et al. (2000) found by fitting first- and second-order autoregressive processes to the principal components of North Atlantic SST in a coupled AGCM-OGCM study with idealized geometry and simplified atmosphere. However, the realistic coupled AGCM-OGCM modeling study by Grötzner et al. (1999) found, using similar methodology to Griffies and Bryan (1997b), significantly shorter predictability times (less than $1 \mathrm{yr}$ ) for the leading mode of SST variability in the midlatitude Northern Hemisphere.

The conceptual theory of SST formation as a red noise process driven by white noise atmospheric forcing (Frankignoul and Hasselmann 1977; Frankignoul 1985) forms the basis of most idealized, stochastic, climate models. Bretherton and Battisti (2000) argue for a predictability time of about 6 months, using a highly idealized, zero-dimensional (OD), coupled energy balance model of the atmosphere and ocean system developed by Barsugli and Battisti (1998, hereafter BB98). However Czaja and Marshall (2000) argue that the BB98 model is not able to reveal the predictability. The only process generating SST anomalies in the model of Barsugli and Battisti is air-sea heat flux. Advection and entrainment of deeper water is not included in their model and may be a fundamental limitation, as they recognized in BB98. This may have implications for both the predictability results and reduction of atmospheric variance found in their model, and formed the major impetus for the current study.

The dominant processes driving midlatitude SST anomalies is a crucial factor in determining their persistence and predictability time. However, the dominant processes are not well known and the situation is complicated by the dependence upon temporal and spatial scale and geographical region. An early review (Namias and Cayan 1981) suggests that air-sea heat exchange, horizontal advection, and turbulent mixing can all play significant roles in generating midlatitude SST anomalies. Yet, the review by Frankignoul (1985) concludes that air-sea heat flux is the dominant mechanism, though the discussion of advection in his section 2 is limited to Ekman currents and mesoscale eddies; large-scale, wind stress curl-driven and nonlinear instability-driven variability in the horizontal currents are not considered. Plenty of research has focused on this problem in the last 16 years. A review is currently being prepared by the first author. The problem remains controversial though most studies highlight the importance of anomalous air-sea heat fluxes driving SST variability (e.g., Cayan 1992; Battisti et al. 1995; Delworth 1996; Kitoh et al. 1999). Other studies suggest that oceanic advection is dominant at least in some regions and on decadal and longer timescales (Delworth et al. 1993; Halliwell 1998; Qiu 2000; Seager et al. 2001).

The primary goal of this paper is to clarify how the inclusion of a deterministic forcing in the mixed layer temperature equation could affect the temperature anomaly predictability time. The motivation is that the discrepancy between the idealized model predictability times (e.g., Bretherton and Battisti 2000) and most fully coupled AGCM-OGCM modeling studies (e.g., Griffies and Bryan 1997b) may be due to more predictable terms driving mixed layer temperature that are missing in the former models. A surrogate for this forcing can be included within the context of the OD model, by adding an arbitrary deterministic forcing to the ocean temperature equation of the BB98 model. In section 2 we quantify the SST anomaly predictability in this model. Although the predictability of anomalous surface geostrophic currents $\mathbf{u}_{g}$ is itself an open question, temperature advection by these currents is a possible physical mechanism for this missing forcing. The advection of anomalous temperature gradients by the time mean flow has been shown by Saravanan and McWilliams (1998) to lead to a novel mechanism of oscillation in a onedimensional (1D) version of the BB98 model. The predictability of SST in that model was analyzed in a follow-up study to the present one (Scott 2003).

There is some evidence, albeit controversial, that the decadal variations in $\mathbf{u}_{g}$ may be predictable. Venzke et al. (2000) forced an OGCM with a prescribed, largescale, wind stress curl pattern and found that a significant portion of the sea level height anomaly response, the second leading EOF that explained $17 \%$ of the variance, lagged the forcing by several years. The wind stress curl pattern was taken from the coupled model study of Latif and Barnett (1994); insofar as their coupled mode is active in nature, the wind anomaly itself may have some predictability. But aside from the wind anomaly predictability, the time delay, which was attributed by Venzke et al. to the gyre adjustment via slow baroclinic Rossby waves, gives some hope to these current anomalies being predictable.

While several idealized studies of midlatitude climate variability have represented the ocean dynamics as linear waves (Jin 1997; Weng and Neelin 1998; Marshall et al. 2001), another perspective is presented in the unique modeling study by Dewar (2001), which emphasizes the importance of nonlinear internal variability of the ocean gyres. Dewar found that the area average advection by geostrophic currents dominated the stochastically driven air-sea heat flux for timescales of a year and longer. This result supports, albeit in a highly idealized modeling study, the data analysis of Qiu (2000). However, these results were not supported by an idealized modeling study using a coupled model with atmosphere (160-km resolution) and ocean (10-km resolution) both represented by two-layer, quasigeostrophic dynamics (Kravtsov and Robertson 2002). Clearly more modeling studies that also resolve the nonlinear gyre dynamics are needed to clarify these issues. Little research has been done in this area, but given that the eddy turnover time in the ocean is about two orders of magnitude longer than that in the atmosphere, one might expect the ocean circulation to have longer predictability times. 
A recent observational data study analyzing the winter season (January-March) terms forcing the ocean mixed layer temperature tendency equation for the $\mathrm{Ku}-$ roshio Extension system revealed that the advection by geostrophic currents may be the dominant source of wintertime, interannual and longer timescale variability in that region (Qiu 2000). This study has been extended here, see section 4, where we analyze all months of the year from October 1992 to September 2001, and address the relative importance of various forcing terms developing monthly SST anomalies in this region.

\section{Description of the stochastically forced, $0 \mathrm{D}$ model of BB98}

\section{a. Background on the BB98 model}

BB98 consider the idealized, 0D, linear model of airsea interactions based upon the stochastic model of Frankignoul and Hasselmann (1977). The atmosphere and ocean are represented by slabs that are characterized by single prognostic variables, $T_{a}(t)$ and $T_{o}(t)$, which are the average temperature anomalies of the atmosphere and ocean. The atmosphere and ocean interact via a heat flux proportional to the local temperature difference,

$$
F=\kappa\left(T_{s}-T_{o}\right),
$$

where $\kappa=\lambda_{s a}\left(\kappa=\lambda_{s o}\right)$ for heat flux into the atmosphere (ocean), and $T_{s}=c T_{a}$ is the surface air temperature anomaly. The small difference between $\lambda_{s a}$ and $\lambda_{s o}$ is due to radiative fluxes. The atmosphere, but not the ocean, also includes a forcing term intended to parameterize the dynamical forcing that is not explicitly represented in the linear model. This forcing is divided into a purely random part $B(t)$ that is white noise in time, and a deterministic part. The deterministic part allows feedback from SST and depends linearly on $T_{o}(t)$, such that the sum of the deterministic, thermodynamic and dynamical forcing of the atmosphere due to SST is $b T_{o}$ where $b$ is their "atmospheric response" parameter. Barsugli and Battisti (1998, p. 482) recommend $0<b<$ 1. (For $b<0$ an anomalously warm ocean tends to cool the atmosphere, which is an unlikely scenario.)

The model equations are derived in BB98 and the nondimensional, time domain versions, are

$$
\begin{aligned}
\frac{d}{d t} T_{a}(t) & =-a T_{a}(t)+b T_{o}(t)+B(t), \\
\beta \frac{d}{d t} T_{o}(t) & =c T_{a}(t)-d T_{o}(t),
\end{aligned}
$$

where $\beta, a$, and $d$ are nondimensional, positive definite, real parameters. The dimensional timescale that nondimensionalizes the time derivative is $\gamma_{a} / \lambda_{s a}$, where $\gamma_{a}$ is the heat capacity of the atmosphere. The parameter values recommended in BB98 are listed in appendix B; see BB98 for further details. Here we simply note that $\beta$ is the ratio of the oceanic to atmospheric heat capacity, and hence we expect

$$
\beta \gg 1
$$

\section{b. The damped harmonic oscillator}

Solving for $T_{o}=x(t)$ we obtain an equation in the form of a stochastically forced, damped, harmonic oscillator (dho),

$$
\ddot{x}+\omega_{o}^{2} x+2 \alpha \dot{x}=\xi,
$$

where $\omega_{o}$ is the natural frequency of the unforced and undamped system,

$$
\omega_{o}^{2}=\frac{a d-b c}{\beta},
$$

$\alpha$ is the damping coefficient,

$$
2 \alpha=\frac{a \beta+d}{\beta} \approx a,
$$

because of the large ocean heat capacity [(3)], and $\xi(t)$ is the purely stochastic, white noise forcing term,

$$
\xi(t)=\frac{c B(t)}{\beta} .
$$

The oscillator is overdamped for $\alpha^{2}>\omega_{o}^{2}$. One can show (appendix A) that this is the case whenever $b c>0$. This implies there is no preferred timescale for $T_{o}$ for positive parameters $a, b, c, d, \beta$. However, a preferred timescale for $T_{o}$ occurs for

$$
b c<-\left(\frac{d^{2}+a^{2} \beta^{2}}{2}\right)<0 .
$$

But this requires either that a warm SST anomaly tends to cool the atmosphere (through some dynamical feedback) or that warm surface air anomalies are associated with cool depth-integrated air temperatures. This is discussed more in appendix A.

\section{c. Red noise process approximation}

In the subsequent analysis for the OD model we made the approximation that for long timescales we can ignore the atmospheric temperature tendency term. As Bretherton and Battisti (2000) mention, the BB98 model then becomes a red noise process. Although the order of the differential equation has been reduced, the results were found not to be singular to this approximation. That is, the analysis with the full ODE [(4)] gave similar results to that with the red noise, approximate ODE,

$$
\dot{x}+\frac{1}{\tau_{\mathrm{dc}}} x=\text { white noise, }
$$

where $\tau_{\mathrm{dc}}$ is the decorrelation time. Thus we only present the simpler, approximate analysis. In appendix $C$ the red noise approximation is justified. 


\section{Predictability of the $0 \mathrm{D}$ model with stochastic and deterministic forcing}

As discussed in the introduction, the predictability times found for the BB98 model are much shorter than the midlatitude SST predictability times found for some coupled AGCM-OGCM studies and the persistence times found in observational data. This motivated looking at the predictability of the stochastically and deterministically forced model.

\section{a. Predictability in a stochastically driven system}

The 6-month predictability time calculated by Bretherton and Battisti (2000) is the decorrelation time of the approximate red noise process. In fact using their parameters (same as BB98 except $\beta=40$ ), one finds a decorrelation time (15) of $\tau_{\mathrm{dc}} \approx 10$ months.

The predictability of the red noise process and the underdamped dho are discussed in a general way in Griffies and Bryan (1997b). For comparison with Bretherton and Battisti (2000), we also calculated the predictability of SST in the BB98 model (again with $\beta=$ 40) based upon the method discussed by Griffies and Bryan. The predictability time $\tau$ is defined as the time period for which the expected mean-square error (MSE) of an optimal forecast reaches a fixed fraction $\gamma$ of the variance (VAR) of the process:

$$
\operatorname{MSE}(\tau)=\gamma \mathrm{VAR} .
$$

(The parameter $\gamma$ is a measure of the choice of forecast skill tolerance.) Using the overdamped harmonic oscillator process [(4)] and the approximate red noise process [(8)] gave similar results. With $\gamma=1 / 2$ predictability defined by (9) gives $\tau \approx 3.7$ and $\tau \approx 3.6$ months, respectively. This method of finding predictability time is more general, and also more stringent for $\gamma=1 / 2$, than using the decorrelation time. It will be used in the next section to find the predictability of SST when deterministic forcing is included in SST evolution [(2)].

\section{b. Predictability with deterministic ocean forcing}

While most terms forcing SST evolution involve atmospheric variables that, in the extratropics at least, are well represented by white noise processes, terms that involve ocean processes are most likely not white noise. In particular, advection by geostrophic currents, and to some extent entrainment at the mixed layer base, involve slower dynamics. It is not immediately clear what noise process, if any, well models these terms. For the sake of generality, here we introduce an unspecified deterministic forcing, with the caveat that it may be unpredictable beyond some timescale. This allows us to develop an expression for the predictability of a simple BB98-type model, but with oceanic forcing. This was done using the full, second-order dho equation for $T_{o}$ [(4)], and also using the red noise approximation, [(8)].
Because the results were similar, only the simpler, red noise approximation is presented. Interpretation of the resulting expression serves to guide the data analysis, for example of section 4, and interpretation of predictability experiments.

It is worth clarifying that of course the deterministic forcing associated with the seasonal cycle enhances predictability albeit in a trivial way. We emphasize that we are, by definition, interested in anomalies about the seasonal cycle and so the deterministic forcing relevant here is of nonseasonal timescale, and more subtle origin.

We include in the ocean temperature tendency equation [(2)] an unspecified, deterministic forcing term, $\beta f_{D}$, that has zero time mean and is analytic (can be represented by a Fourier series). The governing ODE then becomes,

$$
\frac{d T}{d t}=-\frac{T}{\tau_{\mathrm{dc}}}+f,
$$

where we have dropped the subscript on $T_{o}$ since it is the only dependent variable discussed until further notice, $f$ is the total forcing term that now contains a random and deterministic part,

$$
f=f_{R}+f_{D},
$$

with $f_{R}=\xi /(2 \alpha)=c B /(2 \alpha \beta)$ from the red noise ODE approximation for $T[(\mathrm{C} 2)]$.

The optimal forecast has minimum MSE and is the expected value of the solution, $\langle T(t)\rangle$, where the expectation operator is the ensemble average over realizations of the stochastic process $\xi$; see Monin and Yaglom (1971) for a discussion of this operator. Only the stochastic part of the solution contributes to the MSE,

$$
\operatorname{MSE}(t)=\left\langle\left\{\int_{t_{0}}^{t} \exp \left[-\left(\frac{t-u}{\tau_{\mathrm{dc}}}\right)\right] \frac{\xi(u)}{2 \alpha} d u\right\}^{2}\right\rangle .
$$

Using $\langle\xi(u) \xi(v)\rangle=\sigma_{\xi}^{2} \delta(u-v)$, one finds,

$$
\operatorname{MSE}(t)=\frac{\sigma_{\xi}^{2} \tau_{\mathrm{dc}}}{8 \alpha^{2}}\left\{1-\exp \left[-\frac{2\left(t-t_{0}\right.}{\tau_{\mathrm{dc}}}\right]\right\} .
$$

We define the variance to be

$$
\operatorname{VAR}(T) \equiv\left\{[\overline{T(t)-\langle\overline{T(t)}\rangle}]^{2}\right\} .
$$

The time average was introduced because the process is not stationary. For a deterministic forcing of zero time mean, we have $[\overline{T(t)}]=0$. This can be seen by representing $f_{D}$ as a Fourier series. The optimal forecast then has a term that decays exponentially with $\left(t-t_{0}\right)$ and another term that involves the sum of cosine and sine terms, so that the long time mean approaches zero. Thus, $\operatorname{VAR}(T)=\left\{[\overline{T(t)}]^{2}\right\}$. The influence of the initial solution $T\left(t_{0}\right)$ decays exponentially with time and hence only the forced solution contributes to the variance. In fact one can easily show that the variance is simply the sum of the variance due to the stochastic and deterministic forcing $\operatorname{VAR}(T)=\operatorname{VAR}\left(T_{R}\right)+\operatorname{VAR}\left(T_{D}\right)$, with, 


$$
\begin{aligned}
& \operatorname{VAR}\left(T_{R}\right)=\lim _{\left(t-t_{0}\right) \rightarrow \infty}\left\langle\left\{\int_{t_{0}}^{t} \exp \left[-\frac{2(t-u)}{\tau_{\mathrm{dc}}}\right] f_{R} d u\right\}^{2}\right\rangle \\
& =\frac{\tau_{\mathrm{dc}} \sigma_{\xi}^{2}}{8 \alpha^{2}}, \text { and } \\
& \operatorname{VAR}\left(T_{D}\right)=\overline{\left\{\int_{t_{0}}^{t} \exp [-2(t-u)] f_{D} d u\right\}^{2}} .
\end{aligned}
$$

An explicit expression for $\operatorname{VAR}\left(T_{D}\right)$ of course would require that we specify the form of the deterministic forcing. But this is not necessary for drawing conclusions about the implications for predictability. Normalizing the MSE by the variance, we find after some manipulation,

$$
P(t) \equiv \frac{\operatorname{MSE}(t)}{\operatorname{VAR}(T)}=\frac{\left\{1-\exp \left[-\frac{2\left(t-t_{0}\right)}{\tau_{\mathrm{dc}}}\right]\right\}}{1+\frac{\operatorname{VAR}\left(T_{D}\right)}{\operatorname{VAR}\left(T_{R}\right)}} .
$$

This is the main result of the idealized model.

Several important conclusions can be drawn from (11). Of course the deterministic forcing increases the predictability time; this is clear since $\operatorname{VAR}\left(T_{D}\right)$ increases with increasing strength of deterministic forcing $f_{D}$, yet the optimal forecast is not degraded, that is, MSE is unaffected. Furthermore, this expression reveals that the key parameter is the ratio

$$
\chi \equiv \frac{\operatorname{VAR}\left(T_{D}\right)}{\operatorname{VAR}\left(T_{R}\right)} .
$$

It is worth mentioning that the key parameter is not simply the relative strength of the forcing terms, since the response to the forcing, $\operatorname{VAR}\left(T_{R}\right)$, depends upon both the strength of the forcing and the inverse of the decorrelation time. For white noise forcing the response will be nil, $\operatorname{VAR}\left(T_{R}\right)=0$, unless the forcing has infinite variance. Horsthemke and Lefever (1984) suggest that this point is often misunderstood in many fields. Eden and Greatbatch (2002), for example, claim that the BB98 model was forced with unit variance; yet, if this were true the ocean and atmosphere temperature variance would be zero. This is merely a semantic point regarding their work, but the subtlety is emphasized here because it may lead to incorrect conclusions about the relative importance of forcing terms in driving a stochastic dynamical system. Previous studies on the source of SST variability have examined the relative strength of the forcing terms, without regard to their relative decorrelation times. Kitoh et al. (1999) estimated the regression of forcing terms on the SST tendency on a point by point basis, their Fig. 8, to conclude that latent heat flux is the dominant source of variability. This conclusion requires that the forcing terms have similar decorrelation times (which might actually be the case in their study

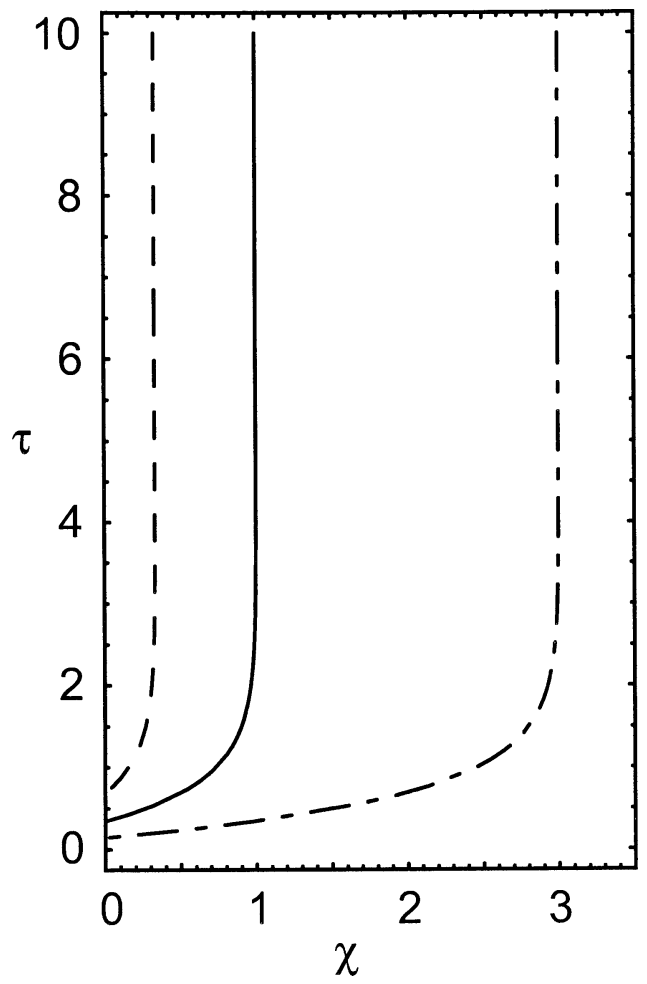

FIG. 1. The predictability time vs relative importance of deterministic and random forcing $\chi \equiv \operatorname{VAR}\left(T_{D}\right) / \operatorname{VAR}\left(T_{R}\right): \gamma=3 / 4$, dashed line; $\gamma=1 / 2$, solid line; $\gamma=1 / 4$, dash-dot line. The predictability time is normalized by the decorrelation time, $\tau_{\mathrm{dc}}$, and thus has units between 6 months and a year, depending upon mixed layer depth. The $y$-intercepts correspond to red noise predictability.

since they only considered surface forcing). They also used a lagged regression between the principal component of SST and the forcing terms as was performed by Delworth (1996). The latter study concludes that anomalous oceanic heat advection plays a lesser role than anomalous surface fluxes in generating the leading modes of winter North Atlantic SST anomalies. But the response to oceanic advection has been underestimated because of the much slower timescale of this forcing, as will be apparent in section 4. A more appropriate comparison is to consider directly the variance driven by each forcing term, as obtained by time integrating the forcing terms.

Another important conclusion is that the influence of deterministic forcing upon predictability is very nonlinear. In Fig. 1 the predictability time is plotted versus $\chi$ for three choices of forecast tolerance $\gamma$. The limit $\chi=0$ corresponds to the case with no deterministic forcing $f_{D}=0$, so that $\operatorname{VAR}\left(T_{D}\right)=0$. As expected, we obtain red noise predictability since (11) reduces to

$$
\frac{\operatorname{MSE}(t)}{\operatorname{VAR}(T)}=\left\{1-\exp \left[-\frac{2\left(t-t_{0}\right)}{\tau_{\mathrm{dc}}}\right]\right\} .
$$

Increasing $\chi$ corresponds to increasing the deterministic forcing and, surprisingly, the predictability time is bare- 
ly affected until an abrupt increase near $\chi=(1 / \gamma-1)$. At this critical value the predictability time based upon $\gamma$ is infinite. In particular, for $\gamma=1 / 2$, (solid line in Fig. 1) for $\operatorname{VAR}\left(T_{D}\right)>\operatorname{VAR}\left(T_{R}\right)$ the predictability time is infinite. This result is of course unrealistic and we must invoke the caveat that the deterministic variations are in practice predictable only for a finite time period. This should be interpreted as implying that the predictability of SST, near $\chi=(1 / \gamma-1)$, is limited by the predictability of the deterministic forcing. The other lines in Fig. 1 are for $\gamma=1 / 4$ and $\gamma=3 / 4$. A qualitatively similar sudden increase holds true, but the critical value depends upon our arbitrary choice of $\gamma$.

A minor point gleaned from (11) is that without the deterministic forcing, the amplitude of the stochastic forcing did not influence the predictability time. With deterministic forcing, this is no longer true.

The previous analysis suggests that it is of interest to SST predictability to determine where upon the $\chi$ axis of Fig. 1 the real ocean lies. A first attempt at this is addressed below via data analysis of the Kuroshio Extension region, selected specifically for its favorable conditions for large oceanic advection.

\section{Kuroshio Extension system mixed layer enthalpy budget}

\section{a. Governing equation and data sources}

Qiu (2000) analyzed the enthalpy budget for the oceanic mixed layer of the Kuroshio Extension region $31^{\circ}-$ $37^{\circ} \mathrm{N}, 141^{\circ} \mathrm{E}-180^{\circ}$, by estimating the terms, averaged over that region, in the mixed layer temperature tendency equation,

$$
\frac{\partial T_{m}}{\partial t}=\mathrm{HF}+\mathrm{VE}+\mathrm{EA}+\mathrm{GA}
$$

where HF $=Q_{\text {net }} /\left(\rho_{o} c_{p} h_{m}\right), \mathrm{VE}=-w_{e}\left(T_{m}-T_{d}\right) / h_{m}$, $\mathrm{EA}=-\mathbf{u}_{e} \cdot \boldsymbol{\nabla} T_{m}$, and GA $=-\mathbf{u}_{g} \cdot \boldsymbol{\nabla} T_{m}$. See Qiu (2000) for a complete description. Here $T_{m}$ is the mixed layer temperature, a proxy for the SST. The mixed layer depth was defined as the depth $h_{m}$ such that the depth-averaged temperature was $1^{\circ} \mathrm{C}$ greater than the water just below,

$$
\frac{1}{h_{m}} \int_{-h_{m}}^{0} T(z) d z-T_{d} \equiv 1^{\circ} \mathrm{C} .
$$

Thus $T_{m}-T_{d}=1^{\circ} \mathrm{C}$ by definition. Monthly upper-ocean temperature data was taken from the Joint Environmental Data Analysis (JEDA) Center of Scripps Institution of Oceanography (White 1995). The entrainment velocity $w_{e}$ was taken from the rate of change of $h_{m}$, and was positive for increasing $h_{m}$ and zero otherwise. The air-sea heat flux, $Q_{\text {net }}$, was taken from the monthly datasets from the National Centers for Environmental Prediction-National Center for Atmospheric Research (NCEP-NCAR) reanalysis (Kalnay et al. 1996; Kistler et al. 2001). The constants $\rho_{o}$ and $c_{p}$ are the seawater reference density and specific heat. The mixed layer
Ekman velocity $\mathbf{u}_{e}=-\hat{\mathbf{k}} \times \boldsymbol{\tau} /\left(\rho_{a} f h_{m}\right)$, where the wind stress $\tau$ was also taken from the NCEP-NCAR reanalysis monthly mean data and $f$ is the Coriolis parameter.

The SST was taken from the dataset described by Reynolds and Smith (1994). For the tendency term, the weekly mean data was used and the central differencing estimate of the time derivative was applied before regridding to monthly means. The time mean geostrophic current $\mathbf{u}_{g}=g \hat{\mathbf{k}} \times \boldsymbol{\nabla} \bar{h} / f$, was calculated from the mean dynamic topography relative to $1000-\mathrm{m}$ depth (Teague et al. 1990). Similar results were found using the mean dynamic topography estimated by Qiu (1995). For the $\mathbf{u}_{g}$ anomalies we used the satellite altimeter data of the TOPEX/Poseidon mission, obtained from the 5-day, $1^{\circ}$ $\times 1^{\circ}$, gridded data of the World Ocean Circulation Experiment (WOCE) satellite data CD-ROM, version 2. The SST data were regridded to 5-day means for the advection calculation, then monthly means estimated.

Because of the finite resolution of the data used, the estimates for the terms in the tendency equation [(12)] ignored the correlation of instantaneous values, that is, between $h_{m}$ and $\boldsymbol{\tau}$, and perhaps most significantly, the eddy heat flux due to unresolved space and timescales (the diffusive flux).

\section{b. Analysis and results}

The seasonal cycle was found to dominate the SST tendency, and the surface heat flux was clearly the dominant source of this variability. This is consistent with the simple scaling assumptions of Gill and Niiler (1973), as well as the findings from ocean weather station data they report. Here, we are interested in the interannual variability so the monthly means were removed to emphasize the anomalies.

Using consecutive monthly data allowed us to estimate the time integral of the $T_{m}$ tendency equation [(12)], providing approximations of the time series of $T_{m}$ and its contributions from each term relative to unknown integration constants. The time integral was approximated via a simple cumulative summation. Applying the integral to the entire length of the record resulted in significant errors. This is to be expected since not only the systematic errors accumulate with time, but also the variance associated with the random errors grows linearly with time (as in a random walk). The time integration is crucial for the analysis however, as argued in section 3. A compromise was reached to remove the monthly anomalies and time-integrate for the first and second half of the record separately. The results are shown in Fig. 2 and some statistics are summarized in Table 1. For the period October 1992 to December 1996 the agreement is very good. Note that the time integral of the anomalies necessarily concludes with a final value of zero, so the integrated forcing and temperature tendency agree by construction in December 1996 and September 2001. The period January 1997 to September 2001 reveals the sensitivity of the method 

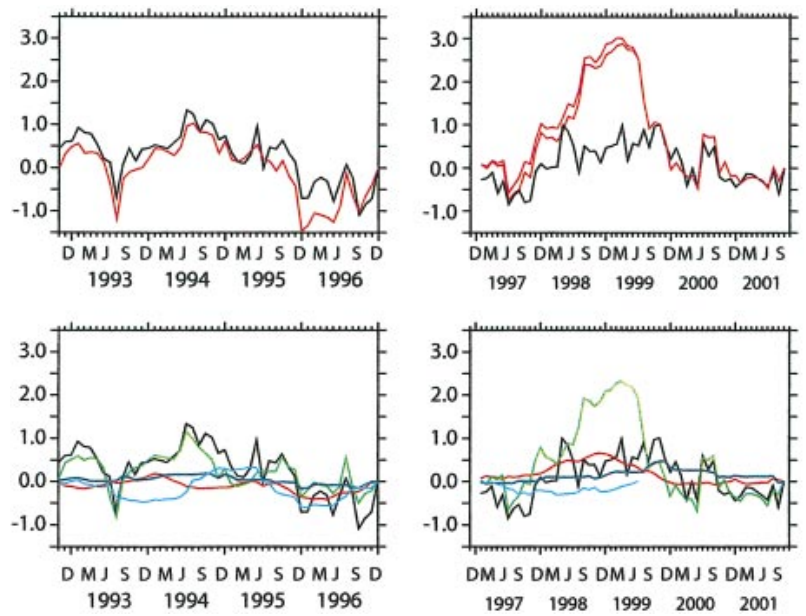

FIG. 2. The time-integrated balance of the monthly anomalies of (12). (left) Oct 1992 to Dec 1996; (right) Jan 1997 to Sep 2001. (top) The time integral of the monthly anomalies of the temperature tendency (black), and the sum of its forcing terms (red). (The extra red line in the upper-right plot shows the influence of the EA, which was only available until May 1999.) The difference between the two curves is due to omitted terms in (12) as well as data and discretization error. (bottom) The components of the forcing terms: $\int^{t} \mathrm{HF} d t^{\prime}$, green; $\int^{t} \mathrm{GA} d t^{\prime}$, red; $\int^{t} \mathrm{VE} d t^{\prime}$, blue; $\int^{t} \mathrm{EA} d t^{\prime}$, light blue. The integration constant is arbitrarily taken as zero. Units are ${ }^{\circ} \mathrm{C}$.

to error. The forcing terms integrate to large deviations that could never occur in nature. The problem is most likely due to errors in the heat flux term (HF), since this term shows a large departure that is unprecedented in the previous $4 \mathrm{yr}$.

The lower two plots in Fig. 2 show the decomposition of the integrated forcing terms, which sum to give the red line in the upper two plots. These time series allow us to associate directly the variability in $T_{m}$ with specific terms. The most striking result was that the air-sea heat flux appeared to be the dominant source of variability, with the other terms serving only to moderate this. The geostrophic advection was the next most significant term, perhaps showing some importance in the winters of 1998 and 1999 and maybe in spring 1996, but otherwise rather insignificant. This runs contrary to observational results for winter months (Qiu 2000) in this region, and the OGCM study by Venzke et al. (2000), who found current anomalies to have a significant impact on the SST field in the Kuroshio Extension region (defined slightly differently in their study).

The variance of each time series in the lower two plots in Fig. 2 is listed in Table 1. The contribution to the total variance from geostrophic advection (GA) varied from only $6 \%$ for the first part of the record to $21 \%$ for the second part. Even for a rather slack choice of forecast tolerance, such as $\gamma=3 / 4$, these results suggest that geostrophic advection does not significantly affect the predictability time, at least on timescales between 1 month and about 4 yr.

Unfortunately the limited quality of the data precludes the possibility of looking for a role for advection on
TABLE 1. Variance, VAR, and correlation with $T_{m}, r^{2}$, and the coefficient of regression upon $T_{m}, \mathrm{RC}$, of each of the time series in the bottom row of Fig. 2. The left columns are for the period Oct 1992 to Dec 1996, While the right columns are for the period Jan 1997 to Sep 2001. All variance values have been normalized by the variance $T_{m}$, which was $0.32\left({ }^{\circ} \mathrm{C}\right)^{2}$ and $0.24\left({ }^{\circ} \mathrm{C}\right)^{2}$ for the earlier and later periods, respectively. The lower half of the table lists the corresponding values after a 7 -month binomial filter was applied. The variance of $T_{m}$ was $0.22\left({ }^{\circ} \mathrm{C}\right)^{2}$ and $0.18\left({ }^{\circ} \mathrm{C}\right)^{2}$ for the earlier and later periods, respectively.

\begin{tabular}{lcccclcc}
\hline \hline Term & HF & VE & EA & GA & HF & VE & GA \\
\hline VAR & 0.47 & 0.03 & 0.24 & 0.06 & 3.3 & 0.07 & 0.21 \\
$r^{2}$ & 0.85 & 0.80 & 0.43 & 0.39 & 0.64 & 0.63 & 0.47 \\
RC & 0.58 & 0.13 & 0.21 & 0.10 & 1.2 & 0.16 & 0.22 \\
VAR & 0.45 & 0.04 & 0.34 & 0.09 & 4.3 & 0.08 & 0.29 \\
$r^{2}$ & 0.87 & 0.89 & 0.52 & 0.54 & 0.68 & 0.70 & 0.53 \\
RC & 0.58 & 0.17 & 0.30 & 0.16 & 1.4 & 0.19 & 0.29 \\
\hline
\end{tabular}

longer timescales, at least with the methods that are employed here. Some hint can be obtained by applying a 7-month binomial filter to the time series of Fig. 2, which reduces the shorter timescale variability. The resulting statistics are also summarized in Table 1. The relative contribution of the advection and entrainment terms to the total variance increases, while the opposite occurred for the surface heat flux term for the first $4 \mathrm{yr}$ (the latter $4 \mathrm{yr}$ saw an increase with filtering, but this portion of the time series is suspect). This indicates that HF makes a relatively greater portion of its contribution on shorter timescales. It is also visually apparent from Fig. 2 that the HF time series is much noisier. As discussed in the introduction, we expect the ocean dynamics to be slower than atmospheric dynamics, so it is not surprising to see that GA has longer timescales than HF. Similarly, the reemergence phenomenon (Alexander and Deser 1995; Alexander and Penland 1996), which forms part of the vertical entrainment (VE) term, is necessarily an interannual signal. The Ekman advection (EA) also had longer timescales, which is surprising since this is driven by wind stress variability.

To emphasize the importance of comparing the timeintegrated forcing terms, as opposed to the forcing terms as previous studies have done, we have repeated the earlier analysis without time integration. The upper plot of Fig. 3 shows the lhs of the SST tendency equation $[(12)]$ in black, and the rhs in red. The agreement is quite reasonable for most of the time period, though note the large error in summer 1998, which may account for the problem in Fig. 2. In the lower plot the rhs is decomposed as in the lower row of Fig. 2. The HF term explains almost all the forcing; in fact the variability in the other terms is barely visible. This is to be contrasted with Fig. 2 in which the time integration has, in effect, low-pass filtered the forcing terms. The highs and lows, on timescales shorter than the SST response time, of the noisy HF term tend to cancel and lead to little contribution to SST formation. These effects can been seen quantitatively by comparing Table 1 with the corresponding values applied to the forcing terms presented 

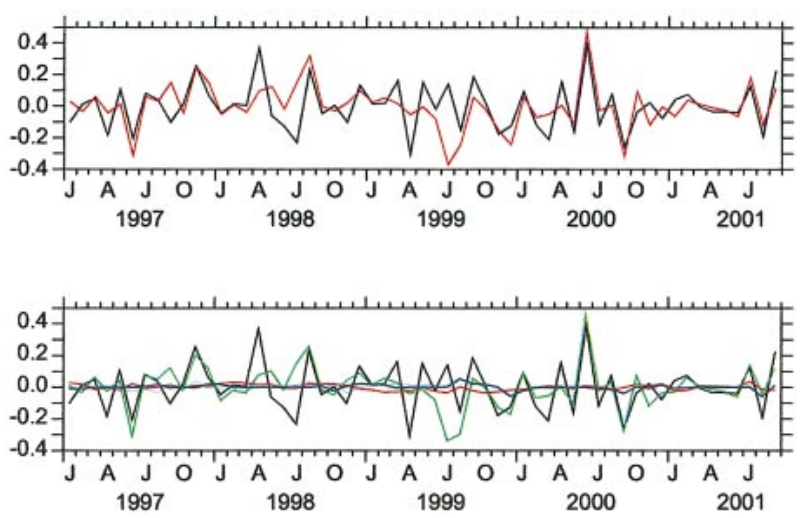

FIG. 3. The monthly anomalies of the temperature tendency and its forcing terms (as before, but no time integration). (top) The lhs of (12) (black), and the rhs of (12) (red). (bottom) The individual components are: HF, green; GA, red; VE blue; EA, light blue. Units are $\mu \mathrm{K} \mathrm{s}^{-1}$.

in Table 2 for the more reliable first half of the record. The relative variance, correlation, and regression coefficients between the slower forcing terms and the SST tendency were very greatly reduced relative to Table 1. The noisier HF was much less altered. A regression analysis between GA and SST tendency would underestimate that obtained using the integrated values by a factor of 5 ( 3 for VE, 2 for EA), but only $10 \%$ for $\mathrm{HF}$. We expect that this difference would be even more exaggerated if higher temporal resolution data were used.

A final point is that the correlation coefficient between the temperature and a given time-integrated forcing term was not a good indication of the importance of the forcing term; the vertical entrainment and surface flux terms had comparable correlation with $T_{m}$ yet the former was clearly the smallest contribution to $T_{m}$ variability and the latter dominated.

\section{Summary and discussion}

We have analyzed some aspects related to predictability of SST anomalies using a linear, coupled, oceanatmosphere model incorporating stochastic and deterministic forcing. Predictability time was based upon the expected time for the mean-square error in the optimal forecast to reach a given fraction $\gamma$ of the total variance of the process. For the OD model of BB98, the SST was found to approach a red noise process for timescales much longer than about 26 days, and to give predictability times of about 4 months $(\gamma=1 / 2)$. The large discrepancy with some coupled GCM results, (e.g., Griffies and Bryan 1997a,b; Saravanan et al. 2000; S. Griffies, T. Delworth, R. Stouffer, and G. Vallis 2001, personal communication), as well as the persistence of SST anomalies in some data studies (Namias and Cayan 1981; Hansen and Bezdek 1996; Sutton and Allen 1997) motivated our investigation of the limitations of the BB98 model. By including a deterministic forcing term
TABLE 2. As in Table 1, but without the time integration, i.e., the monthly anomalies of each term in the $T_{m}$ tendency [(12)].

\begin{tabular}{lccccccc}
\hline \hline Term & HF & VE & EA & GA & HF & VE & GA \\
\hline VAR & 0.55 & 0.01 & 0.06 & 0.02 & 0.78 & 0.01 & 0.01 \\
$r^{2}$ & 0.70 & 0.50 & 0.43 & 0.14 & 0.57 & 0.19 & 0.01 \\
RC & 0.52 & 0.05 & 0.11 & 0.02 & 0.50 & 0.02 & 0.001 \\
\hline
\end{tabular}

in the ocean temperature equation, a simple model for the predictability was developed that may be useful for guiding data analysis and interpreting predictability experiments.

The observational data study of the oceanic mixed layer enthalpy budget of the Kuroshio Extension system by Qiu (2000) was extended here by time-integrating the monthly anomalies of each term in the budget. This allowed us to decompose the mixed layer temperature anomaly time series into its contributions from each term. Unfortunately the errors in the data limited us to consider time periods about $4 \mathrm{yr}$ in length. Most significantly we found that the variance associated with the surface heat flux to be the dominant component of variability for monthly to 4-yr timescales. The temperature advection and entrainment were only a minor source of variability, so predictability is unlikely to be affected by these terms. This supports the use of the $0 \mathrm{D}$ model of BB98 on these timescales, even in this highly turbulent region of the ocean.

Unfortunately this result also leaves as unexplained the important discrepancies between, on the one hand, SST predictability times found with some GCMs and the observed persistence of SST anomalies, and on the other hand, some other GCMs and the FrankignoulHasselmann theory of SST formation. Several factors must be stressed about the longer SST predictability times of Griffies and Bryan (1997b). They also found that the EOF patterns naturally sorted the variability according to predictability time. Perhaps the average SST in the rectangular region we defined here is much less predictable than the leading EOFs. Furthermore, insofar as the thermohaline circulation (THC) variability is relevant to SST predictability, we may expect differences between the North Atlantic and North Pacific Oceans. This is suggested by the fact that when the THC was less active the predictability of SST (and other variables) was about one-half its peak predictability of 5 yr (Griffies and Bryan 1997b). This may also be relevant for understanding the discrepancy with Northern Hemisphere results of Grötzner et al. (1999). Furthermore, the ocean gyre and THC in the study of Griffies and Bryan (1997b) had decorrelation times of around $10 \mathrm{yr}$. This timescale of variability is beyond what we could address with the data here. Finally, the analysis here of SST averaged over the Kuroshio Extension region is not well suited to capturing propagating signals that were observed in the North Atlantic (Hansen and Bezdek 1996; Sutton and Allen 1997).

A positive contribution of this study is the extension 
of a simple model that can be used to evaluate the SST predictability when deterministic forcing is present. Two important results from this model are: 1) that the key parameter affecting the predictability is the relative SST variance associated with stochastic and deterministic forcing, and 2) that we expect the predictability to increase rapidly near a critical level of this parameter. The former should be contrasted with previous studies that have addressed the strength of the various forcing mechanisms without regard to their decorrelation times. The latter suggests a clue for the discrepancy between various GCM studies in that we may expect sensitivity to model parameters near the critical value. To make further progress in the SST predictability problem we require either more accurate upper-ocean and surface marine datasets, and/or another method to estimate the variance associated with the various forcing mechanisms. The possibility of using a damping term that will cause the influence of earlier errors to exponentially decay with time is currently under investigation.

Acknowledgments. The predictability calculations benefited greatly from conversations with Stephen Griffies. Geoff Vallis first drew my attention to the discrepancy between idealized and coupled GCM predictability results and encouraged me to try to understand this discrepancy. Thanks also to Joe Barsugli and an anonymous reviewer for their very thorough and thoughtful reviews, which helped improve this work significantly, especially with the data analysis section. Funding from Natural Science and Engineering Research Council of Canada and Princeton University are gratefully acknowledged. Reynolds SST data was provided by the NOAA-CIRES Climate Diagnostics Center, Boulder, Colorado, from their web site at http:// www.cdc.noaa.gov/. (If using this data, please note the definition of the averaging period.) Altimeter data from the World Ocean Circulation Experiment Satellite Data CD-ROM, version 2.0, was provided by the Jet Propulsion Laboratory from their web site at http:// podaac.jpl.nasa.gov/cdrom/woce2_topex/.

\section{APPENDIX A}

\section{Preferred Timescales in the 0D Model}

The characteristic equation for the dho [(4)] is

$$
\lambda^{2}+2 \alpha \lambda+\omega_{0}^{2}=0,
$$

where $\lambda$ is the eigenvalue. Thus real eigenvalues (overdamped solutions) are associated with $\alpha^{2}>\omega_{0}^{2}$. Using definitions (5) and (6), this inequality becomes, after some manipulation,

$$
\left(\frac{a}{2}-\frac{d}{2 \beta}\right)^{2}>-\frac{b c}{\beta} .
$$

Given that $\beta$ is large, recall (3), clearly for the case where $b c>0$ the above condition is met and the dho
TABLE B1. Nondimensional parameter values of the BB98 model.

\begin{tabular}{cccccccc}
\hline \hline Parameter & $a$ & $b$ & $c$ & $d$ & $\beta$ & $\omega_{o}^{2}=\frac{a d-b c}{\beta}$ & $\alpha=\frac{a \beta+d}{2 \beta}$ \\
\hline Value & 1.12 & 0.5 & 1 & 1.08 & 20 & 0.188361 & 0.587 \\
\hline
\end{tabular}

is necessarily overdamped. This implies that there is no preferred timescale for this case.

Furthermore, for nongrowing solutions to (4), we require a real, natural frequency for the undamped and unforced system, $\omega_{0}^{2}>0$. In terms of the BB98 model (1) and (2) this is the case for $a d>b c$, as follows immediately from the definition of $\omega_{o}[(5)]$. One can find the conditions for a preferred timescale in $T_{a}$ and $T_{o}$ by looking for resonance, that is, the conditions of a local maximum at positive frequencies in the corresponding power spectra density (PSD). With this restriction of nongrowing solutions, we found resonance will occur for $T_{o}$ only when

$$
b c<-\left(\frac{a^{2} \beta^{2}+d^{2}}{2}\right)<0 .
$$

Physically this implies either a warm SST anomaly tends to cool the atmosphere (through some dynamical feedback) or that warm surface air anomalies are associated with cool depth-integrated air temperatures. The predictability was examined under these conditions but the results were not presented because the physical relevance was not clear. Note that the "advective limit" discussed by Webster (1981) could provide a mechanism whereby the dynamical response of the atmosphere tends to cool, via cold advection, the air over a warm SST anomaly in a westerly mean flow. However it is unlikely that the cool advection could dominate the diabatic warming. Furthermore, the canonical correlation data analysis of Zorita et al. (1992) did not find this mode of atmospheric response to SST to be significant over the North Atlantic.

\section{APPENDIX B}

\section{Parameters of BB98 Model}

The parameters of the BB98 model can be found in their Table 1. These are reproduced in Table B1 here for convenience.

The dimensional timescale in the BB98 model, implicit in (1) and (2), is based upon the heat capacity of the atmosphere $\gamma_{a}$ and the effective damping coefficient $\lambda_{s a}$. The recommended values (see BB98 Table 1) are $\gamma_{a}=10^{7} \mathrm{~J} \mathrm{~m}^{-2} \mathrm{~K}^{-1}$ and $\lambda_{s a}=23.9 \mathrm{~W} \mathrm{~m}^{-2} \mathrm{~K}^{-1}$, which gives a timescale of about 5 days.

\section{APPENDIX C}

\section{Approach to Red Noise}

Here we find the precise conditions required for the 
dho process (4), with purely stochastic forcing, to approach the red noise process (8). Clearly we require the acceleration term, that is, the first term in (4), to be negligible while the other terms remains significant. This can be made more precise by working with the Fourier transform of the nondimensional dho:

$$
-\epsilon^{2} \hat{T}_{o}+2 i \frac{\alpha}{\omega_{o}} \epsilon \hat{T}_{o}+\hat{T}_{o}=\frac{\hat{\xi}}{\omega_{o}^{2}},
$$

where we have normalized by $\omega_{o}^{2}$ and introduced the parameter $\epsilon \equiv \sigma / \omega_{o}$, where $\sigma$ is the nondimensional frequency. Clearly for small $\epsilon$ the acceleration term becomes small. But to approach the red noise process with significant $\tau_{\mathrm{dc}}$ (as opposed to passing directly to white noise, that is, negligible $\tau_{\mathrm{dc}}$, we also require $2 \alpha / \omega_{o} \geq$ $O\left(\epsilon^{-1}\right)$. This is only possible for the very strongly overdamped case $\alpha \gg \omega_{o}$.

For these conditions we introduce the approximate ocean temperature $T_{r} \approx T_{o}$ that satisfies,

$$
\hat{T}_{r}=\frac{\hat{\xi}}{\omega_{o}^{2}\left(1+2 i \frac{\alpha}{\omega_{o}} \epsilon\right)},
$$

or in the time domain, we have the red noise process ODE,

$$
2 \alpha \dot{T}_{r}+\omega_{o}^{2} T_{r}=\xi .
$$

Note the nondimensional decorrelation time $\tau_{\mathrm{dc}}=2 \alpha / \omega_{o}^{2}$. Because of the approximation (6), this is very similar to the decorrelation time that arises from simply dropping the acceleration term in the time domain:

$$
\tau_{\mathrm{dc}}=\frac{a \beta}{a d-b c}=\frac{a}{\omega_{o}^{2}} .
$$

The power spectra density, $\operatorname{PSD}\left(T_{r}\right)$ of this red noise process is,

$$
\operatorname{PSD}\left(T_{r}\right)=\left\langle\hat{T}_{r} \hat{T}_{r}^{*}\right\rangle=\frac{\left\langle\hat{\xi} \hat{\xi}^{*}\right\rangle}{\omega_{o}^{4}+4 \alpha^{2} \sigma^{2}}
$$

One can easily show that the PSD of the stochastically forced dho,

$$
\left\langle\hat{x} \hat{x}^{*}\right\rangle=\frac{\langle\hat{\xi} \hat{\xi} *\rangle}{\left(\omega_{o}^{2}-\sigma^{2}\right)^{2}+(2 \alpha \sigma)^{2}},
$$

approaches $\operatorname{PSD}\left(T_{r}\right)$ for $\epsilon^{2} \ll 1$ and $\epsilon 2 \alpha / \omega_{o} \geq O(1)$.

What range of parameters satisfy the above criteria? $\epsilon^{2} \ll 1$ implies $\sigma^{2} \ll \omega_{o}^{2}$ so that in dimensional terms, using the parameters given by BB98 (see appendix B),

$$
\omega \equiv \frac{\lambda_{s a}}{\gamma_{a}} \sigma \ll \frac{\lambda_{s a}}{\gamma_{a}} \omega_{o} \approx \frac{1}{26 \text { days }} .
$$

We must also check if the second criterion $\epsilon 2 \alpha / \omega_{o} \geq$ $O(1)$ is realistic. This criterion implies that

$$
O(\epsilon)=\frac{\omega_{o}}{2 \alpha} \ll 1
$$

For the parameters given by BB98,

$$
\frac{\omega_{o}}{2 \alpha}=\left(\frac{a d-b c}{\beta}\right)^{1 / 2}\left(\frac{\beta}{a \beta+d}\right) \approx 0.16,
$$

so that it is reasonable to declare $\omega_{o} /(2 \alpha) \ll 1$.

Note that for even longer times, that is, long relative to the decorrelation time,

$$
\omega^{-1} \gg \frac{\gamma_{a}}{\lambda_{s a}} \tau_{\mathrm{dc}} \approx 5 \text { months, }
$$

both $T_{a}$ and $T_{o}$ approach white noise processes,

$$
\begin{aligned}
& T_{o}(t)=\frac{\xi(t)}{\beta_{o}^{2}}=\text { white noise } \\
& T_{a}(t)=\frac{b}{a} T_{o}(t)+\frac{1}{a} B(t)=\text { white noise. }
\end{aligned}
$$

\section{REFERENCES}

Alexander, M. A., and C. Deser, 1995: A mechanism for the recurrence of wintertime midlatitude SST anomalies. J. Phys. Oceanogr., 25, 122-137.

_ , and C. Penland, 1996: Variability in a mixed layer ocean model driven by stochastic atmospheric forcing. J. Climate, 9, 24242442 .

, I. Bladé, M. Newman, J. R. Lanzante, N.-C. Lau, and J. D. Scott, 2002: The atmospheric bridge: The influence of ENSO teleconnections on air-sea interaction over the global oceans. $J$. Climate, 15, 2205-2231.

Barsugli, J. J., and D. S. Battisti, 1998: The basic effects of atmosphere-ocean thermal coupling on midlatitude variability. $J$. Atmos. Sci., 55, 477-493.

Battisti, D. S., U. Bhatt, and M. A. Alexander, 1995: A modeling study of the interannual variability in the wintertime North Atlantic. J. Climate, 8, 3067-3083.

Bond, N. A., and D. Harrison, 2000: The Pacific decadal oscillation, air-sea interaction and central North Pacific winter atmospheric regimes. Geophys. Res. Lett., 27, 731-734.

Bretherton, C. S., and D. S. Battisti, 2000: An interpretation of the results from atmospheric general circulation models forced by the time history of the observed sea surface temperature distribution. Geophys. Res. Lett., 27, 767-770.

Cayan, D. R., 1992: Latent and sensible heat flux anomalies over the northern oceans: Driving the sea surface temperature. J. Phys. Oceanogr., 22, 859-881.

Czaja, A., and J. Marshall, 2000: On the interpretation of AGCMs response to prescribed time-varying SST anomalies. Geophys. Res. Lett., 27, 1927-1930.

Delworth, T. L., 1996: North Atlantic interannual variability in a coupled ocean-atmosphere model. J. Climate, 9, 2356-2375.

__ S. Manabe, and R. J. Stouffer, 1993: Interdecadal variations of the thermohaline circulation in a coupled ocean-atmosphere model. J. Climate, 6, 1993-2011.

Dewar, W. K., 2001: On ocean dynamics in midlatitude climate. $J$. Climate, 14, 4380-4397.

Eden, C., and R. Greatbatch, 2002: Prospects for the decadal prediction of the North Atlantic oscillation. Geophys. Res. Lett., in press.

Frankignoul, C., 1985: Sea surface temperature anomalies, planetary waves, and air-sea feedback in the middle latitudes. Rev. Geophys., 23, 357-390.

_, and K. Hasselmann, 1977: Stochastic climate models. Part II: Application to sea-surface temperature variability and thermocline variability. Tellus, 29, 284-305.

Gill, A. E., and P. P. Niiler, 1973: The theory of the seasonal variability in the ocean. Deep-Sea Res., 20, 141-177. 
Griffies, S. M., and K. Bryan, 1997a: Predictability of the North Atlantic multidecadal climate variability. Science, 275, 181-184 , and - 1997b: A predictability study of simulated North Atlantic multidecadal variability. Climate Dyn., 13, 459-487.

Grötzner, A., M. Latif, and A. Timmermann, 1999: Interannual to decadal predictability in a coupled ocean-atmosphere general circulation model. J. Climate, 12, 2607-2624.

Halliwell, G. R., 1998: Simulation of North Atlantic decadal/multidecadal winter SST anomalies driven by basin-scale atmospheric circulation anomalies. J. Phys. Oceanogr., 28, 5-21.

Hansen, D. V., and H. F. Bezdek, 1996: On the decadal anomalies in North Atlantic sea surface temperature. J. Geophys. Res., 101, 8749-8758.

Horsthemke, W., and R. Lefever, 1984: Noise-Induced Transitions: Theory and Applications in Physis, Chemistry, and Biology. Springer-Verlag, $318 \mathrm{pp}$.

Jin, F.-F., 1997: A theory of interdecadal climate variability of the North Pacific ocean-atmosphere system. J. Climate, 10, 18211835.

Kalnay, E. M., and Coauthors, 1996: The NCEP/NCAR 40-Year Reanalysis Project. Bull. Amer. Meteor. Soc., 77, 437-471.

Kistler, R. E., and Coauthors, 2001: The NCEP-NCAR 50-year reanalysis: Monthly means. CD-ROM and documentation. Bull. Amer. Meteor. Soc., 82, 247-267.

Kitoh, A., T. Motoi, and H. Koide, 1999: SST variability and its mechanisms in a coupled atmosphere-mixed layer ocean model. J. Climate, 12, 1221-1239.

Kravtsov, S., and A. W. Robertson, 2002: Midlatitude ocean-atmosphere interaction in an idealized coupled model. Climate Dyn., 19, 693-711.

Kushnir, Y., and I. M. Held, 1996: Equilibrium atmospheric response to North Atlantic SST anomalies. J. Climate, 9, 1208-1220.

Latif, M., and T. Barnett, 1994: Causes of decadal climate variability over the North Pacific and North America. Science, 266, 634637.

- and Coauthors, 1998: A review of the predictability and prediction of ENSO. J. Geophys. Res., 103, 14 375-14 393.

Marshall, J., H. Johnson, and J. Goodman, 2001: A study of the interaction of the North Atlantic Oscillation with ocean circulation. J. Climate, 14, 1399-1421.

Monin, A. S., and A. M. Yaglom, 1971: Statistical Fluid Mechanics: Mechanics of Turbulence. Vol. 1. MIT Press, 769 pp.

Namias, J., and D. R. Cayan, 1981: Large-scale air-sea interactions and short-period climatic fluctuations. Science, 214, 869-876.

Palmer, T. N., 1996: Predictability of the atmosphere and oceans: From days to decades. Decadal Climate Variations: Dynamics and Predictability, D. L. Anderson and J. Willebrand, Eds.,
NATO ASI Series I: Global Environmental Change, Vol. 44, Springer, 83-155.

Qiu, B., 1995: Variability and energetics of the Kuroshio Extension and its recirculation gyre from the first two-year TOPEX data. J. Phys. Oceanogr., 25, 1827-1842.

- 2000: Interannual variability of the Kuroshio Extension system and its impact on the wintertime SST field. J. Phys. Oceanogr., 30, 1486-1502.

Reynolds, R. W., and T. M. Smith, 1994: Improved global sea surface temperature analyses using optimum interpolation. J. Climate 7, 929-948.

Saravanan, R., and J. C. McWilliams, 1998: Advective ocean-atmosphere interaction: An analytical stochastic model with implications for decadal variability. J. Climate, 11, 165-188.

_ , G. Danabasoglu, S. C. Doney, and J. C. McWilliams, 2000 Decadal variability and predictability in the midlatitude oceanatmosphere system. J. Climate, 13, 1073-1097.

Scott, R., 2003: Predictability of SST in an idealized, one-dimensional, coupled atmosphere-ocean climate model with stochastic forcing and advection. J. Climate, 16, 323-335.

Seager, R., Y. Kushnir, N. H. Naik, M. A. Cane, and J. Miller, 2001 Wind-driven shifts in the latitude of the Kuroshio-Oyashio Extension and generation of SST anomalies on decadal timescales. J. Climate, 14, 4249-4265.

Sutton, R. T., and M. R. Allen, 1997: Decadal predictability of North Atlantic sea surface temperature and climate. Nature, 388, 563567.

Teague, W. J., M. J. Carron, and P. J. Hogan, 1990: A comparison between the Generalized Digital Environmental Model and Levitus climatologies. J. Geophys. Res., 95, 7167-7183.

Venzke, S., M. Münnich, and M. Latif, 2000: On the predictability of decadal changes in the North Pacific. Climate Dyn., 16, 379392.

Webster, P. J., 1981: Mechanisms determining the atmospheric response to sea surface temperature anomalies. J. Atmos. Sci., 38, $554-571$.

Weng, W., and J. D. Neelin, 1998: On the role of ocean-atmosphere interaction in midlatitude interdecadal variability. Geophys. Res Lett., 25, 167-170.

White, W. B., 1995: Design of a global observing system for gyrescale upper ocean temperature variability. Progress in Oceanography, Vol. 36, Pergamon, 169-217.

Yuan, X., and D. G. Martison, 2000: Antarctic sea ice extent variability and its global connectivity. J. Climate, 13, 1697-1717.

Zorita, E., V. Kharin, and H. von Storch, 1992: The atmospheric circulation and sea surface temperature in the North Atlantic area in winter: Their interaction and relevance for Iberian precipitation. J. Climate, 5, 1097-1108. 